\title{
Agent Compatibility and Coalition Formation: Investigating Two Interacting Negotiation Strategies
}

\author{
Carlos Merida-Campos and Steven Willmott \\ Universitat Politècnica de Catalunya, Software Department, E-08034 Barcelona, \\ Spain \\ $\{$ dmerida, steve\}@lsi.upc.edu
}

\begin{abstract}
This paper focuses on Coalition Formation paradigm as a market mechanism. Concretely, Coalition Formation occurs as part of a wider open world and may occur many times during the lifetime of a population of agents. This fact can in some circumstances be exploited by agents to re-use partial existing coalition and social relationships over time to improve Coalition Formation efficiency. The aim of the work is to analyze the dynamics of two concrete rational behaviors (Competitive and Conservative strategies) and, in particular, to investigate how agents in a heterogeneous population cluster together across multiple Coalition Formation episodes and varying tasks. Preliminary resuls are also shown regarding how agents playing distinct strategies interact with one another.
\end{abstract}

\section{Introduction}

One branch of research on economics investigates how specific market situations force a certain behavior in their participants. A common example of this phenomenon is Smith's Invisible Hand, under which, in competitive market scenarios, traders are forced to set the prices where the supply meets the demand [13]. This specific situation does not appear too often in its pure form, and traders must often choose in what could be a large space of different pricing strategies.

The main agent-based research in the Economic area, focuses on studying strategy interactions and dynamics given a certain market. In this paper we focus on the related question of strategies and dynamics in market mechanism scenarios where Coalition Formation is required. Coalition Formation is a problem which has been extensively studied from Multi-Agent Systems perspective. The problem centers on finding subsets of agents from a general population to form groups which can most effectively carry out a particular task. The area includes research on a variety of different fronts, including problems such as finding core allocations of payoff between the members of a coalition [1], finding stability properties between coalition structures [7], finding solutions to a problem in a cooperative way [5], or finding solutions to a coalition problem in a competitive way, where coalitions compete amongst themselves for a payoff [12]. 
In the market mechanism we suggest in the present work, coalition formation occurs as part of a wider open world and may occur many times during the lifetime of a population of agents. As shown in [12] this fact can in some circumstances be exploited by agents to re-use partial coalition and social relationships over time to improve Coalition Formation efficiency. Such a broader perspective however raises interesting questions for coalition formation environments. This paper goes further in that direction analyzing the dynamics of two concrete rational behaviors, i.e. a Competitive Strategy and a Conservative Strategy within such an environment. These strategies are common examples of rational behavior in economic markets. There are, indeed more complex strategies, but, as argued in this paper these two strategies already prove sufficient to create two differentiated types of dynamics.

This type of continuously running market environment requiring coalitions reflects a large number of real world scenarios. In this context we aim to answer the following questions:

- To what extent do specific strategies affect the type of coalitions formed?

- To what extent do specific strategies affect the stability properties of the system?

- How do different agents with different strategies affect each other when they interact?

- Which strategies benefit agents and the general population more in which situations?

The paper is structured as follows: Section 2 explains the concrete market mechanism we are using, the Iterative RFP Coalition Formation method as well as examples to illustrate the method. Section 3 explains the agent based system designed to model the Iterative RFP Coalition Formation Method. Section 4 explains the characteristics of the strategies we are testing in this paper, and analyzes them from a theoretical perspective. Section 5 explains the experimental setup and the results obtained to underpin the theoretical analysis and to provide some new information. Section 6 covers related work and finally, sections 7 and 8 provide conclusions and ongoing / future work repsecitvely.

\section{Iterative RFP Coalition Formation Method}

As the basis for experimentation, analysis in this paper adopts a model of worlds based on Request For Proposal (RFP from now on) scenarios. This model was first studied by [11], and further explored in [12]. In this environment, an entity or entities regularly issues a call for tender to provide specific goods or services with certain characteristics. Providers compete between themselves (either individually or in cosortia - coalitions). Providers and/or coalitions bidding for a particular call are ranked according to some evaluation function based on metric evaluated over their skills for the task and receive a payoff according to their placement in the ranking. 
There are many existent real systems that follow the RFP type procedures such as public building projects, competitive tender for government contracts or even collaborative research project grants. RFP environments can also be seen as emerging market opportunities in an economy, with individual calls for tender representing new opportunities for profit. Such system are characterized as follows:

- Agents, or groups of agents compete for a given goal.

- The best agents, or groups of agents are rewarded with the award of the contract to carry out the task and its subsequently payoff.

- Some systems, may also have policies for rewarding 2 nd, $3 \mathrm{rd}$, 4 th etc. ranked agents / groups of agents.

- The process repeats over time.

- The objective agents are competing for varies over time - with different CFPs issued corresponding to functionally different tasks. In this way, an agents or a group of agents that were very competent for a certain goal, could become weak for a different one.

- Groups could be dynamic and might change depending on the market situation.

- Agents are individual utility maximizers. And share the same preference, that is payoff maximization.

\section{Problem Definition and Agent Based Models}

For the purposes of this paper, the problem is formalized in the following way: In every game $g$ there is a task $T_{g}$ that is defined by a set of $K$ tuples. Each tuple represents an skill and its corresponding demanding value for the named task:

$$
T_{g}=\left\{\left\langle s k_{0}, T_{g 0}\right\rangle,\left\langle s k_{1}, T_{g 1}\right\rangle, \ldots,\left\langle s k_{K}, T_{g K}\right\rangle\right\}
$$

Every agent $A_{i}$ in the population has a certain capability in each of the $K$ skills that task $T_{g}$ is defined with: $\left\{\left\langle s k_{0}, A_{i 0}\right\rangle,\left\langle s k_{1}, A_{i 1}\right\rangle, \ldots,\left\langle s k_{K}, A_{i K}\right\rangle\right\}$.

A coalition $C_{j}$ is a a set of one or more agents $\left\{A_{x}, \ldots, A_{z}\right\}$. A Coalition is also endowed with a certain capability in each of the $K$ skills that task $T_{g}$ is defined with: $\left\{\left\langle s k_{0}, C_{j 0}\right\rangle,\left\langle s k_{1}, C_{j 1}\right\rangle, \ldots,\left\langle s k_{K}, C_{j K}\right\rangle\right\}$.

Each skill capability of a coalition will be an aggregated value from all the skill values of agents in the coalition. The concrete aggregation function we use is:

$$
C_{j p}=\max _{\forall q: A_{q} \in C_{j}} A_{q p} \quad \text { Having } 0 \leq p \leq K .
$$

We could consider each skill as a necessary subtask for performing task $T_{g}$. In this way, by using the aggregation function shown in equation 1 , the agent in a coalition which is the best fit for performing a certain subtask will be in charge of it.

Among the different possibilities for aggregating agent skills in a coalition, we have chosen the one as we consider is a reasonable metaphor of many real 
coalitional processes. For example, if we consider a consortium of partners participating in a call for proposals, each member of the consortium will be representative of a certain part of the proposal, and normally is the partner best fitted for that part of the work.

Coalition $C_{j}$ is endowed with a certain score $\operatorname{scr}\left(C_{j}, T_{g}\right)$. This score will be negative if the coalition is non competent in all the skills for performing the task $\left(\exists p:\left(0 \leq C_{j p}<T_{g p}\right)\right)$, and will be positive otherwise. More concretely, the functions we use for the case of existence of non competent skills in the coalition is:

$$
\operatorname{scr}\left(C_{j}, T_{g}\right)=-\# s k_{p}:\left(0 \leq C_{j p}<T_{g p}\right)
$$

When Coalition $C_{j}$ is competent in every skill $\left(\forall p:\left(0<T_{g p} \leq C_{j p}\right)\right)$, the function we will use is:

$$
\operatorname{scr}\left(C_{j}, T_{g}\right)=\sum_{p=0}^{K} C_{j p}-T_{g p}
$$

As we can see in Equation 2, the score of a coalition with some non competent skill (non competent coalition), is the negative value of the count number of non competent skills. In this way, the more skills in which the coalition is not competent, the lower its score will be.

In Equation 3, we can see that the score of a coalition, competent in every skill (competent coalition) is the sum of extra value in every requested skill. In both equations, we can see that only those skills with value higher than 0 count for their evaluation. Those with values equal to 0 are ignored. This represents the fact that some tasks will not need a certain skill to be performed, and so the degree of ability of a coalition in that skill will not be taken into account for its evaluation.

There is non-linear mapping from coalition score to coalition payoff, as in our model, the payoff of a coalition will depend not only on its score but also on the scores of other coalitions. All coalitions are ordered by decreasing order of score, and will be priced according to its rank with an exponentially decreasing amount from the initial one. Agents within a coalition spread the coalition payoff evenly. The concrete payout function we use is:

CoalReward $($ rank $)= \begin{cases}\text { MaxAmount } / 2^{\text {rank-2 }}, & \text { for the last competent coal. } \\ \text { MaxAmount } / 2^{\text {rank-1 }}, & \text { for the other competent coals }\end{cases}$

Among the different possibilities for doing the mapping, we have chosen this concrete exponential function for of two reasons: first, because this function has the property that, independently of the number of competent coalitions, the total amount of money spread will always be MaxAmount. This is an interesting property in order to compare the economic behavior of different populations. The second reason is because this represents a power law distribution of wealth for which there is empirical evidence from Real Economies (see [6]). This function 
creates a rich set of possible scenarios in which coalitions optimize their trade-offs between score and size, growing in size only when it is valuable to do so.

For every game, a subset of agents will be asked at random about an action to take towards its membership in a coalition. The choices that an agent has are:

- Stay in the coalition.

- Stay in the coalition optimizing it by firing (expelling) one or more members.

- Leave the coalition in order to join a different one.

- Leave the coalition in order to replace one or more agents in a different one.

- Create a new coalition.

When an agent's decision involve firing or replacing an agent, this action will be submitted to the coalition who will evaluate it. For getting the action approved and executed, it must be approved for more than the half of the members of the coalition affected, otherwise the action is rejected and not performed. In that case that an agent is fired or replaced, it automatically becomes the only member of a brand new coalition.

When agents are requested to perform an action they are able to submit as many proposals as they want, if none of them is accepted the agent will remain in the same coalition where it was.

Agents are farsighted in the sense that they know the score and payoff values of any action the agent wants to consider prior to its submission.

\section{Conservative and Competitive Strategies}

In this paper we have implemented two basic strategies to participate in the RFP Coalition Formation market. Agents who choose a conservative strategy will make decisions on which coalition to join based on the payoff this coalition is expected to win. Agents who choose a competitive strategy will make decisions on which coalition to join based on the score that this coalition will have. Both strategies are myopically rational, as when an agent using some of those strategies is asked to make a choice, it will count with all the information available at that instant of time, concretely, they will have the information on potential payoff and score of any coalition they could create by doing any of the 5 possible actions defined in the previous section, but will not count on the possible reactions of the other agents after its decision has been performed.

Both strategies are also individually rational. In the case of conservative strategy, it makes sense to choose the most profitable coalition at a certain time, expecting that the situation will not change until the end of the game. For the case of competitive strategy, it makes sense to join the coalition with highest score, as in spite of the payoff could be worse than in other coalitions, a new member could be attracted by this growing score coalition and make it grow in the ranking an gain a higher payoff. In other words, for the case of conservative strategy, the agent expects to get the maximum payoff at any time, while for competitive strategy, the agent invests for a future better payoff as a side effect of being in a highly competent coalition. 


\subsection{Theoretical Dynamics of Competitive Population}

Competitive agents will try to be in a coalition with the highest possible score. At the same time, coalitions of Competitive agents will accept joining proposals, optimizations or replacements proposed as long as they improve the coalition score $^{1}$. This behavior ensures that, as for a given task during the process of Coalition Formation, each movement of an agent from coalition $A$ to coalition $B$ that takes place involves an improvement in the score of coalition $B$. It will always happens that the score of $A$ is less than the score of $B$ after the movement, otherwise the agent would not had had any motivation to move there. Thus the maximum score of the best coalition in our setup will be monotonically increasing during the periods of task stability (games in which a certain task do not change). Agents will not create coalitions with more members that skills, as the most competitive coalition will have an "expert" agent per skill. Having more agents would mean having redundant agents in the coalition, hence needlessly reducing payoff to existing members. As the coalition size is limmited by that maximum size, the score of the best coalition will stabilize at a certain point. The same will happen with the score of the second best coalition, and so on. In this way, the system will become stable in a certain configuration of coalitions.

\subsection{Theoretical Dynamics of Pure Conservative Population}

Conservative agents will try to be in a coalition with the highest possible per agent payoff. At the same time, coalitions of Conservative agents will accept joining proposals, optimizations or replacements proposed as long as they improve the coalition per agent score ${ }^{2}$. This behavior ensures that during the process of coalition formation, each movement of an agent from coalition $A$ to coalition $B$ that takes place involves an improvement in the score and payoff per agent of coalition $B$ (otherwise, the coalition would not have had any motivation to accept the new member). Payoff per agent in coalition $A$ prior the movement is less or equal than in coalition $B$ after the movement, but, as a difference of the previous strategy, we cannot say that the score of $A$ is less than the score of $B$. This is because an agent might prefer to be in an average scored coalition with few members to split the payoff, than in a top scored coalition with many members to split the payoff.

In this environment, coalitions will not tend to grow in the same way as in the previous strategic environment. Sizes of the coalitions will be determined by the concrete skill distribution among the population, the requirements of the task and the payoff function. More concretely, the growing possibilities will be determined by the score differences from coalitions in the ranking. Let $A_{1}$ be an agent, $C_{1}$ be a coalition and $\backslash C_{1}$ the rest of coalitions competing in a certain moment. Ranking of $C_{1}$ is $\operatorname{rank}\left(C_{1}, \backslash C_{1}\right)$, its size is $\# C_{1}$, and its payoff

\footnotetext{
${ }^{1}$ As a secondary criterium of acceptance, we use the size of the coalition, i.e. if the coalition score is the same, we will propose/accept when the number of agents is smaller than in the origin/original coalition.

${ }^{2}$ Same secondary criterium of acceptance than in competitive strategy.
} 
is $\operatorname{pay}\left(\operatorname{rank}\left(C_{1}, \backslash C_{1}\right)\right) / \# C_{1}$. We can claim that $C_{1}$ will never grow in size as long as:

$$
\nexists A_{1}: \operatorname{pay}\left(\operatorname{rank}\left(C_{1} \cup A_{1}\right)\right) /\left(\# C_{1}+1\right)>\operatorname{pay}\left(\operatorname{rank}\left(C_{1}\right)\right) / \# C_{1}
$$

Then the difficulty of a coalition has in growing depends on payoff function. As the payoff function we are using in our model is monotonically decreasing as a function of the ranking of the coalition, in order to have a higher payoff in coalition $C_{1} \cup A_{1}$, the following must be true:

$$
\operatorname{rank}\left(C_{1} \cup A_{1}\right)>\operatorname{rank}\left(C_{1}\right)
$$

From this, we can see that the possibilities of a coalition to grow up in size also depend on how difficult what is stated in Condition 6 is ${ }^{3}$. As a matter of fact, the difficulty of condition 6 to hold for any arising coalitions, depends on the skill distribution of agents, and on the size of coalitions, in such a way that the smaller the coalition, the easier condition 6 will be fulfilled. The Influence of coalition size is explained as follows:

In a coalition with no redundant agents, each agent is giving the maximum value to one or more skills. If the coalition has few agents, its members will each have more skills to be responsible for. If agents of a population have the same total sum of skill values $\left(\sum_{p=0}^{K}\left(A_{i p}\right)\right)$, there will be many agents with different skill values in the distribution that could improve the coalition score by taking responsibility of many more skills than if the coalition were large and each skill would be responsibility of a certain "expert" agent. In this way, by having more chances of increasing coalition score by raising the value of more skills, we will have also more chances of rising the coalition rank.

The stability analysis in this population is more complex than in competitive population. The system will be stable once agents have reached Pareto optimality, and Nash equilibrium. This situation is made more complex by different factors. An important one is that if coalitions do not need to grow too much to be competent, we will have many small coalitions, and as we have seen in the analysis of the importance of the coalition size, for the case of small coalitions it will be easier for agents to find profitable coalitions outside their current coalition. This could create a continuous movement of agents from coalition to coalition.

\section{Experiments}

To examine the dynamics of the strategies explained and validate the theoretical analysis, a range of simulation experiments were conducted. Each simulation run consists of a set of a fixed number of model iterations, where agents follow the

\footnotetext{
${ }^{3}$ Note that it could happen that joining more than one agent to the coalition $\left(C_{1} \cup\right.$ $A_{1} \cup \ldots A_{n}$ ) we could improve the coalition enough for making it profitable for all the agents on it, but given our model, agents do not take decisions in a coordinated manner.
} 
Iterated $R F P$ protocol explained in section 2, for solving a number of different tasks.

In order to visualize the relationships established between agents in the experiments, we have used Pajek [3]. Graph figures represent the relationships created between agents throughout a series of games of one or more experiments. Each node is an agent, and a link represents a collaboration that existed when the coalition was evaluated in a certain game. Agents collaborate when they are together in a competent coalition. In this way, a coalition is represented by a clique of connections among the agents in the coalition. In order to ease the visual analysis edges are colored depending on the frequency of the relationship; the more often a collaboration happens, the darker will be the line. The graph is represented using Kamada-Kawai algorithm implemented in Pajek that place nodes in a close position when they are connected with links of relative high value. In our case, agents appear close to each other when they have had frequent relationships. Throughout the rest of the paper, we will call these graphs collaboration graphs.

\subsection{Experimental Set-Up}

We have used a variety of configurations and parameterizations in order to be able to check the statistical validity of outcomes. The underlying configuration for the experiments was:

- A static population of 100 agents, with abilities randomly distributed across 10 different skills. Each skill of an agent may be assigned a positive integer score or zero. Agents are each assigned 200 skill points randomly distributed across their skills.

- A set of 100 different Tasks. Each one with requiring a total of 100 skill points distributed randomly across the same 10 different skills.

- Each task is issued during 1000 games. And each game picks 25 agents at random to make a choice on their coalition preference (and hence potentially adapt their coalition according to the schemes defined in the previous section).

- Every competent coalition is subsequently ranked and rewarded according to function 4. The concrete MaxAmount value used for this function is 100 . This represents the total amount that will be spread in each game among the competent coalitions.

During the experiments, we monitor in the Collaboration Graph each relationship established at the end of every round. We also monitor the payoff data for each one of the agents, as well as the coalition sizes.

\subsection{Competitive Behavior Experiments}

In these experiments, we use a population of 100 agents following the Competitive behavior strategy. Figure 1(a) shows the collaboration graph of the population in just one experiment, and figure 1(b) represents the aggregated results 
among the set of 100 experiments with different tasks to fulfill. In the first figure we can appreciate 30 vertex out of the existent 100, this is because the algorithm places vertices close to each other when relationships between them are very strong (in our case, strength values means frequent relationships), that means that some coalitions of agents repeat very frequently over time. We also see few residual low frequency relationships of some agents with some of other clusters. Those two facts verifies the analysis performed in section 4.1. There we drawn the conclusion that a population of agents using Competitive strategy will converge to a stable state where at a certain point of the process, there will be no movement of agents among coalitions. In the graph, we observe that some clusters of nodes repeat very frequently, these are the structures created after the optimization process has finished. Residual relationships (relationship with low frequency) belong to the period in which the game has not reached the stability. At that period agents are still in an optimization process. Another fact that validates in the theoretical analysis is the size of coalitions, that among the whole set of experiments, tends to be equal to the number of requested skills by the task, having normally an expert agent on charge of every skill. We computed the mean size of coalitions for the case of 100 experiments obtaining 8.2, which is very close to the mean value of requested skills among the different tasks that is 8.5 .

The second figure shows us that even changing the task, we can distinguish clear clusters of agents. This is explained by the following fact: Competitive agents will create coalitions that maximize the value of all the skills requested by a task. In order to do that, they will create coalitions as big as necessary, having an "expert" agent for each requested skill, i.e. an agent which only contribution will be to have the biggest value in the coalition for an specific skill. The only difference in coalitions created for different tasks will be in the not inclusion of "expert" Agents for those skills not requested in a concrete task, while keeping the rest of the agents working together, hence keeping frequent relationships in the main part of the coalition, as in average, tasks issued usually have no more than 2 non-required skills (that vary from task to task) out of 10 skill.

\subsection{Conservative Behavior Experiments}

In these experiments, we use a population of 100 agents following the conservative behavior strategy. Figure 1(c) shows the collaboration graph of the population of just one experiment, and figure $1(\mathrm{~d})$ represents the aggregated results among the set of 100 experiments with different tasks to fulfill.

In the first figure we can appreciate how collaboration is not concentrated in clusters, instead it is spread in many different combinations of agents. This suggests the correctness of the analysis performed in section 4.2 , where we stated that a conservative population will optimize the size of coalitions and if the size turns out to be small given the concrete skill setup, there will be a high degree of dynamism in the coalitions. We can appreciate this in our experiments, where the average size of coalitions formed is 2.3 , and the distribution of nodes indicates that agents do not form durable coalitions. However, some nodes in the 

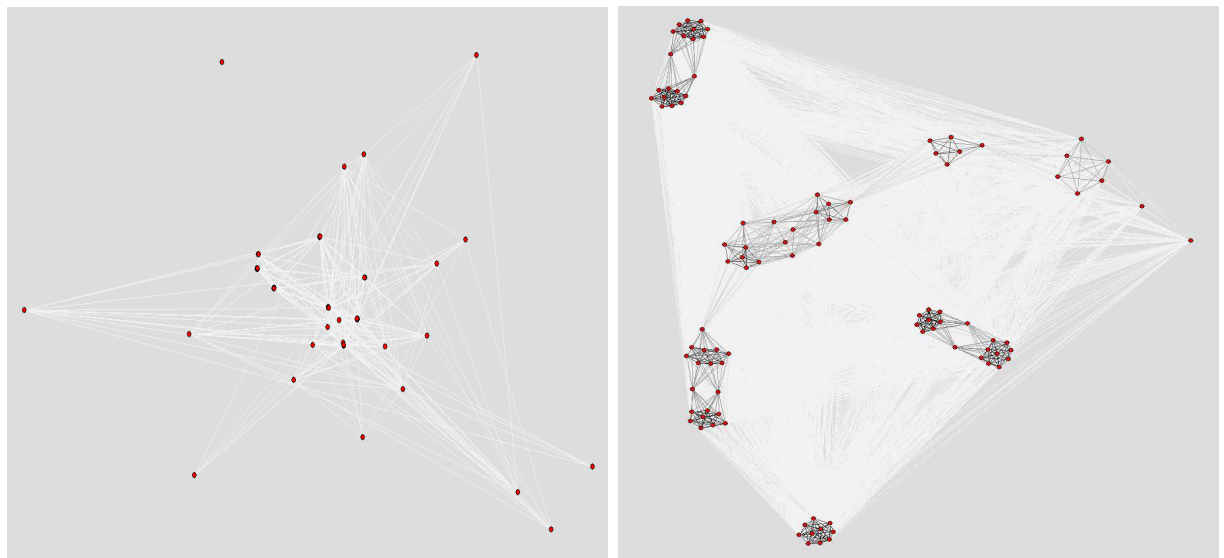

(a) 1 Experiment Result pure competitive (b) 100 Experiments Result pure competipopulation tive population
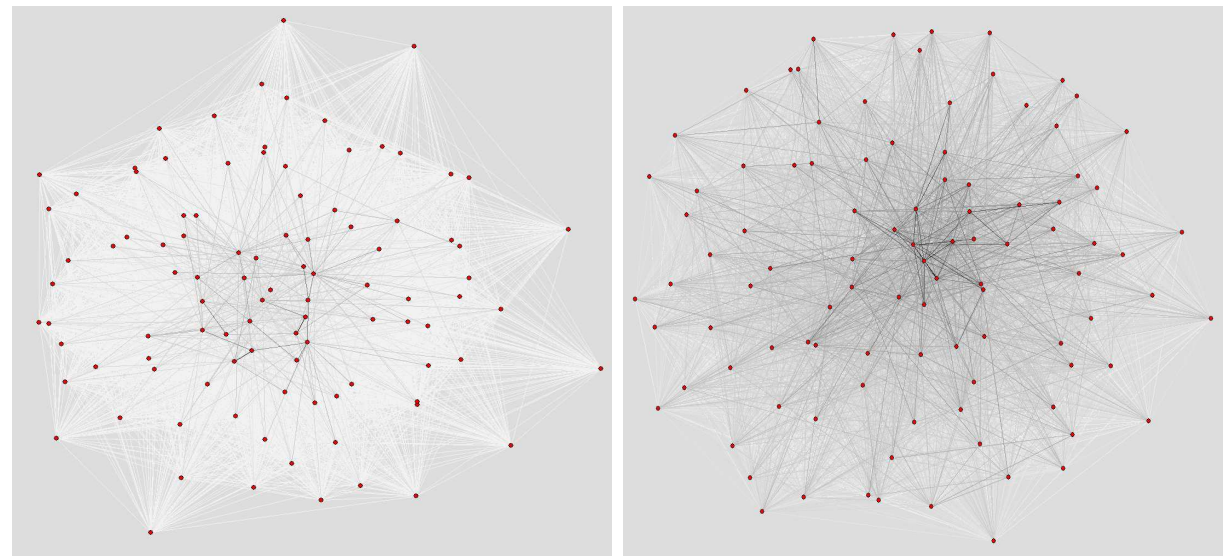

(c) 1 Experiment Result pure conservative (d) 100 Experiments Results pure conserpopulation vative population
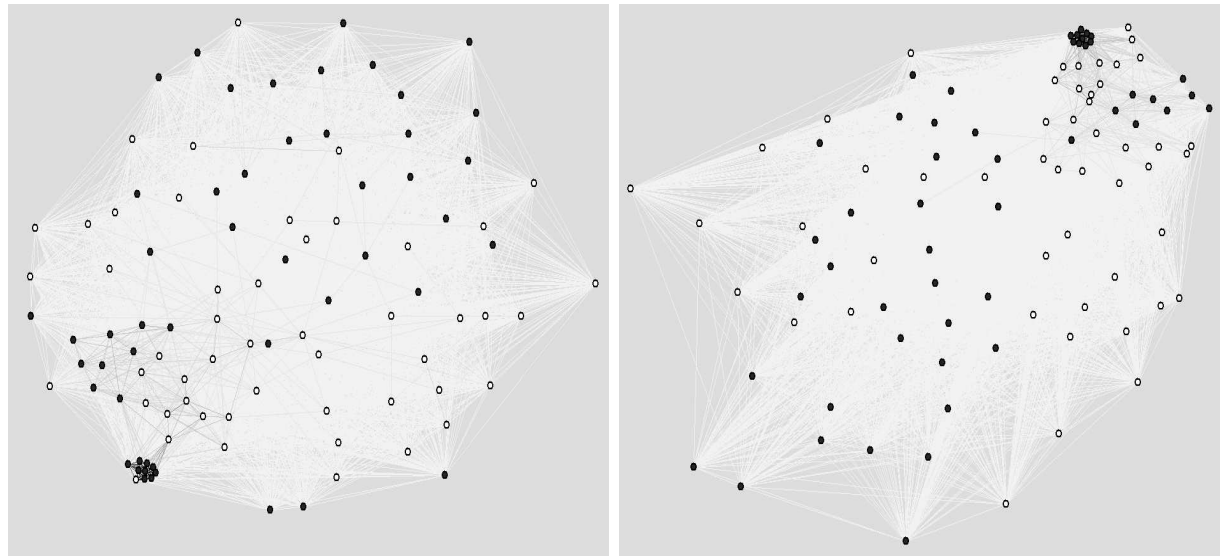

(e) 1 Experiment Result mixed population (f) 100 Experiments Results mixed population

Fig. 1. Collaboration Graphs of different populations settings 
center of the graph show slightly more frequent connections between them. This suggests that in this environment there are successful agents that appear more often in competent coalitions, and also, that these agents have some preferential attachments with other nodes with which they can create small coalitions with good scores, and so, it happens that they meet each other more frequently.

By observing figure 1(d) representing the aggregation of the data obtained across all the experiments, we can see that it keeps the same concentric structure, indicating that successful properties of central agents are kept. By the dark color in the edges, we can see that although conservative agents spread their collaboration, they usually cooperate with the same wide range of agents.

\subsection{Mixed Strategies Dynamics Experiments}

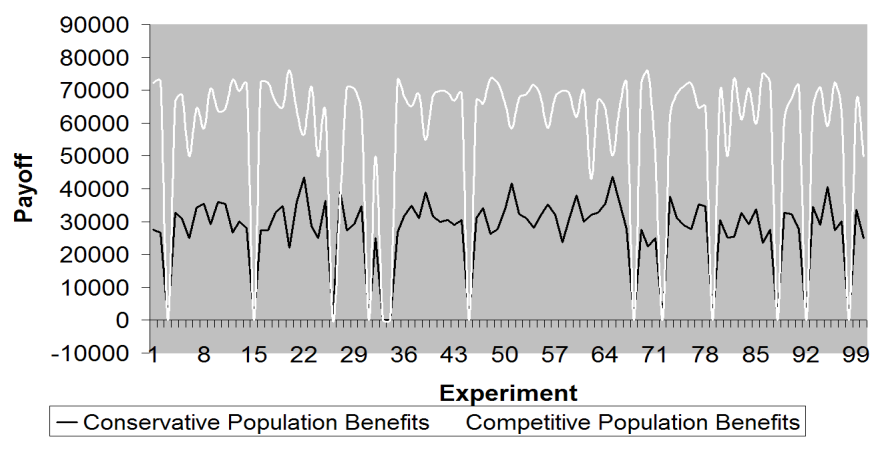

Fig. 2. Payoff gained by each population throughout 100 different tasks.

In these experiments, we mix 50 agents using the competitive strategy with 50 agents using conservative strategy. In order to create comparable results when playing two populations together, we create a symmetric population of 50 duplicated skills vectors. Figure 1(e) shows the collaboration graphs of the population in just one experiment. Figure 1(f) on the right hand side represents the results among the set of 100 experiments with different tasks to fulfill. To differentiate members of each population in the graph, nodes representing conservative agents are in black color, and nodes representing competitive agents are in white color.

In the first graph we can see how a group with a majority of competitive agents establishes itself in the experiment, and show almost pure endogamic relationships. Apart from this, we observe that the rest of agents spread their collaboration with many different members of any population type. The average coalition size in this experiment is 5.9. This indicates that competitive agents boost the competitivity in the games, and large coalitions created by those agents become attractive also to conservative agents, as once the coalition has a certain competitive degree it becomes more profitable than small coalitions created by other conservative agents. 
The figure showing results throughout the 100 experiments reflects a very similar situation as in the one experiment graph. Both figures have a shape that reflects the mixture between clustering into frequent groups and spreading among many different ones. We still appreciate a cluster of competitive agents, and very close to that cluster, reflecting frequent collaboration with the elements of the cluster, we have a set of conservative agents. Those agents will not stay in the cluster coalitions as frequently as competitive agents, because they are probably tempted by other coalitions offering less score and higher payoff, but they are probably good enough to be accepted in the coalitions when those coalitions become the most profitable option for a conservative agent at a certain time.

In terms of profit, as we can see in figure 2, competitive population clearly outperforms conservative population. This is explained by the fact that competitive agents will be the founder creators of the most competitive coalitions and will remain loyal. On the other hand, conservative agents that get attracted by a successful high score coalition, will join it, but the competitive agents will likely remain the a majority. Moreover, conservative agents in a high score high profit coalition might leave it seeking apparent opportunities in the market of smaller coalitions with higher benefits. This turns out often to be the wrong decision as smaller coalitions are more dynamic and subject to change (see section 4.2 for an explanation of this fact).

\section{Related Work}

There is other relevant work in literature that tackles the heterogeneity of populations as a key point of the research. The most representative is [5] which studies how diversity within the agent population impacts on the quality of the coalitions that emerge. Conforth et. al. create a dynamic organization framework in which heterogeneity turns out to be a crucial element for problem solving tasks. In this work, heterogeneity is represented upon different initial values of agent's parameters such as their connectivity (interaction links), trading strategies and initialization states. In our model, all agents know each other but are distinguished by different values in a fixed set of skills. This fact characterizes every individual by intrinsic properties that can be complementary, and have different dynamics from those used in the cited work. Our agents, are also differentiated by the type of strategy they use to negotiate. Another interesting example on the use of heterogeneity in coalition formation processes is to model heterogeneous preferences. Some examples of this are [2] which models different preferences between leisure and work, and [4] which provides a method that considers the possibility of different evaluation functions to coalition structures. Apart from that concrete work, the majority of models existent in the literature of MAS in Coalition Formation use heterogeneity in the agent populations, at least in the initial conditions, however, those differences are not the main focus of their research, they just constitute one more variable in the model.

From the point of view of the protocol used (RFP), our work is related to [11]. However, Kraus et. al. have radical differences in its use. Firstly, in these models, 
a number of tasks are issued and agents propose coalitions (from scratch) that are accepted or rejected by the affected members. In order to motivate agents to form a coalition, a discount factor is used. In our case we just have one Task at a time for which all the population compete for, but not only one will get priced. In order to motivate agents to form good coalitions, we use a decreasing payment function.

We share many characteristics with economic models, such as [2]. Some important characteristics are: the iterative nature of the processes, the evaluation of the structures episodically, and similar type of rational behavior in the agents, however there is an important difference that is the evaluation function. The evaluation function applied by Axtell et. el. (Cobb-Douglass) rewards a coalition (or a firm) independently of the rest of existing coalitions. In our case the reward of a coalition is partly dependant on its score, and on the score of the rest of the existent coalitions.

Traditionally Coalition Formation problems have been tackled as a one off event. In our work we seek to go beyond this to consider what may happen in environments over time. Other important work in the same line includes $[9,10,1]$.

\section{Conclusions}

From the analytical and experimental work presented, the following conclusions, applicable to the Iterative RFP domain, are drawn:

- Competitive strategy (score maximizing) outperforms conservative strategy (payoff maximizing) when symetric populations are played against one another. This provides a hint as to why competitivity emerges in certain societies to dominate other conservative behaviours.

- A pure population of agents running the competitive strategy, will reach an equilibirum state with respect to specific tasks in the environment.

- A pure population of agents running the competitive strategy will tend to create coalitions of size equal to the number of requested skills.

- A pure population of agents running the conservative strategy will tend to create small size coalitions.

- In a pure population of agents running the conservative strategy, there is a inversely proportional relationship between the size of coalitions and the degree of dynamism.

Lastly, the long term aim of this work is to analyse the clustering structure of agents within an RFP population - and investigate how this affects performance. To this end, Collaboration Graph representation seems to present a useful method to analyze properties of the Coalition Formation process in such long running scenarios.

\section{Ongoing and Future Work}

Ongoing and future work includes a deeper research on the conditions under which competitivity dominates conservationism. 
It will be interesting to investigate the individual characteristics that make agents extraordinary, and extract the important patterns that are exploitable with certain strategies such as those shown in the current paper.

It will be also of significant value to study the stability of results from a formal game theoretic perspective, as well as the game theoretic properties of the strategies we study.

Finally, we are investigating the use of other large scale network analysis techniques to investigate deeper our domain. Such techniques include Clique Overlapping, and t-core distribution.

\section{References}

1. T. Arnold and U. Schwalbe. Dynamic coalition formation and the core. Journal of Economic Behavior \& Organization, 49:363-380, 2002.

2. R. Axtell. The emergence of firms in a population of agents: Local increasing returns, unstable nash equilibria, and power law size distributions. Working Paper 3, Center on Social and Economic Dynamics, Brookings Institution, 1999.

3. V. Batagelj and A. Mrvar. Pajek-program for large network analysis. http://vlado.fmf.uni-lj.si/pub/networks/pajek/.

4. P. Cailou, S. Aknine, and S. Pinson. A multi-agent method for forming and dynamic restructuring of pareto optimal coalitions. In Proceedings of the 1st conference on Autonomous Agents and Multi-Agent Systems, AAMAS'02, 2002. Bologna, Italy.

5. D. Cornforth, M. Kirley, and T. Bossomaier. Agent heterogeneity and coalition formation: Investigating market-based cooperative problem solving. In Proceedings of the 3rd conference on Autonomous Agents and Multi-Agent Systems, AAMAS'04, 2004. New York, USA.

6. A. Dragulescu and V. M. Yakovenko. Exponential and power-law probability distributions of wealth and income in the united kingdom and the united states. Computing in Economics and Finance 2002 125, Society for Computational Economics, July 2002.

7. D. D. Gatti and C. D. Guilmi. Financial fragility, industrial dynamics and business fluctuations in an agent based model. paper presented ad the conferenceWild@Ace 2003, Turin, Italy, October 3-4, 2003.

8. D. D. Gatti, C. D. Guilmi, E. Gaffeo, G. Giuioni, M. Gallegati, and A. Palestrini. A new approach to business fluctuations: heterogeneous interacting agents, scaling laws and financial fragility, 2003.

9. M. Klusch and A. Gerber. Dynamic coalition formation among rational agents. IEEE Intelligent Systems, 17(3):42-47, 2002.

10. H. Konishi and D. Ray. Coalition formation as a dynamic process. Journal of Economic Theory, 110:1-41, 2003.

11. S. Kraus and O. S. ang G.Tasse. Coalition formation with uncertain heterogeneous information. In Proceedings of the 2nd Conference on Autonomous Agents and Multi-Agent Systems, AAMAS'03, 2003. Melbourne, Australia.

12. C. Merida-Campos and S. Willmott. Modelling coalition formation over time for iterative coalition games. In Proceedings of the $3 \mathrm{rd}$ conference on Autonomous Agents and Multi-Agent Systems, AAMAS'04, 2004. New York, USA.

13. V. L. Smith. An experimental study of competitive market behavior. The Journal of Political Economy, 70:111-137, 1962. 